\title{
Tanshinone IIA enhances bystander cell killing of cancer cells expressing Drosophila melanogaster deoxyribonucleoside kinase in nuclei and mitochondria
}

\author{
HAIYANG JIANG ${ }^{1}$, LEI ZHAO ${ }^{2}$, XIAOSHEN DONG ${ }^{1}$, ANNING HE $^{3}$, CAIWEI ZHENG ${ }^{4}$, \\ MAGNUS JOHANSSON $^{5}$, ANNA KARLSSON ${ }^{5}$ and XINYU ZHENG ${ }^{1,3}$ \\ ${ }^{1}$ Department of Breast Surgery, The First Affiliated Hospital, China Medical University; \\ ${ }^{2}$ Center of Experiment Technology and Medical Research, China Medical University; ${ }^{3}$ Laboratory 1, Cancer Institute, \\ China Medical University, Shenyang, Liaoning, P.R. China; ${ }^{4}$ Brandeis University, Waltham, MA, USA; \\ ${ }^{5}$ Department of Laboratory Medicine, Karolinska Institute, Stockholm, Sweden
}

Received May 6, 2015; Accepted June 15, 2015

DOI: 10.3892/or.2015.4102

\begin{abstract}
Heterologous expression of the Drosophila melanogaster multi-substrate deoxyribonucleoside kinase (Dm-dNK) increases the sensitivity of cancer cells to several cytotoxic nucleoside analogs. Thus, it may be used as a suicide gene in combined gene/chemotherapy treatment of cancer. To further characterize this potential suicide gene, we constructed two retroviral vectors that enabled the expression of $D m$-dNK in cancer cells. One vector harbored the wild-type enzyme that localized to the nucleus. The other vector harbored a mitochondrial localized mutant enzyme that was constructed by deleting the nuclear localization signal and fusing it to a mitochondrial import signal of cytochrome $c$ oxidase. A thymidine kinase-deficient osteosarcoma cell line was transduced with the recombinant viruses. The sensitivity and bystander cell killing in the presence of pyrimidine nucleoside analogs (E)-5-(2-bromovinyl)-2'-deoxyuridine and $1-\beta$-D-arabinofuranosylthymine were investigated. Tanshinone IIA is a constituent ofDanshen; a traditional Chinese medicine used in the treatment of cardiovascular diseases. This study also looked at the influence of Tanshinone IIA on the bystander effect and the underlying mechanisms. We showed that sensitivity of the osteosarcoma cell line to the nucleoside analogs and the efficiency of bystander cell killing were independent of the subcellular localization of Dm-dNK. The enhanced effect of tanshinone IIA on the bystander effect was related to the increased expression of $\mathrm{Cx} 43$ and $\mathrm{Cx} 26$.
\end{abstract}

Correspondence to: Professor Xinyu Zheng, Department of Breast Surgery, The First Affiliated Hospital, China Medical University, 155 North Nanjing Street, Shenyang, Liaoning 110001, P.R. China E-mail: xyzheng126@126.com

Key words: tanshinone IIA, nucleoside kinase, nucleoside analog, bystander effect, suicide gene

\section{Introduction}

In suicide gene therapy, a foreign gene is introduced into tumor cells and the expression of the gene converts a non-toxic prodrug into a lethal drug, such as nucleoside kinases that phosphorylate cytotoxic nucleoside analogs (1). The most extensively studied suicide genes include the herpes simplex virus type-1 thymidine kinase (HSV-1 TK) with ganciclovir (GCV) as a prodrug, and the cytosine deaminase (CD) of Escherichia coli, which converts the non-toxic antifungal agent fluorocytosine (5-FC) into 5-fluorouracil (5-FU) $(2,3)$. Cells expressing HSV-1 TK phosphorylate GCV, which is then incorporated into nuclear DNA during DNA replication and repair. The incorporation of GCV terminates DNA elongation and causes cell death (4). The killing of adjacent cells due to the transportation of phosphorylated GCV via gap junctions, known as the 'bystander effect', is important for successful suicide gene therapies $(5,6)$.

Previously, we identified a multi-substrate deoxyribonucleoside kinase (Dm-dNK) from the fruit fly Drosophila melanogaster and evaluated the possible use of this enzyme as a suicide gene in vitro and in vivo (7-11). Dm-dNK phosphorylates a broad range of substrates including analogs of both purine and pyrimidine nucleosides, and exhibits a higher activity than previously studied nucleoside kinases $(12,13)$. When expressed in human cells, $D m$-dNK localizes to the nucleus through a C-terminal nuclear localization signal (8). We previously performed mutagenesis of the nuclear localization signal and investigated the effect of heterologous expression of a cytosolic Dm-dNK on cancer cells (10). There were no differences between cytosolic $D m$-dNK and nuclear $D m$-dNK in regards to enzyme activity, cellular sensitivity to nucleoside analogs, or bystander cell killing.

Tanshinone (Tan) IIA is a fat-soluble and pharmacologically active ingredient of Danshen; a traditional Chinese medicine used in the treatment of cardiovascular diseases. It is isolated from the rhizome of a Chinese herb Salvia miltiorrhiza $(10,14)$. Previous studies have shown that Tan IIA possessed not only anti-inflammatory (15) and antioxidant 
properties, but also anticancer activities in cell culture and animal carcinogenesis models $(16,17)$. Tan IIA can restore connexin (Cx) 43 by inhibiting the elevated miR-1 expression in ischemic and hypoxic cardiomyocytes $(18,19)$. Connexins constitute a family of structurally related transmembrane proteins, including $\mathrm{Cx} 43$ and $\mathrm{Cx} 26$, which connect two adjacent cells via gap junctional intercellular communication (GJIC) (20). Dysfunction of connexins may lead to defects in cell proliferation, differentiation and localization, which may be correlated with tumorigenesis (21-23). Some studies have shown that GJIC is directly involved in the bystander effect, by which adjacent cells are sensitized to drug treatment during gene therapy (24-27). Considering all these facts, Tan IIA may influence the bystander effect of cancer cells expressing $D m$-dNK by regulating the expression of Cx43 and Cx26.

In the present study, we further characterized $D m$-dNK as a suicide gene when the enzyme was expressed in different subcellular compartments. We compared the cytotoxicity and the bystander effects of the nucleoside analogs (E)-5-(2-bromovinyl)-2'-deoxyuridine (BVDU) and $1-\beta$-D-arabinofuranosylthymine (araT) when the enzyme was expressed in either the nuclei or the mitochondria. We showed that a recombinant $D m$-dNK with a mitochondrial targeting signal localized to the mitochondria, retained high enzymatic activity. The cells expressing mitochondrial $D m$-dNK and nuclear $D m$-dNK had similar sensitivities to the nucleoside analogs and similar bystander effects. The subcellular localization of $D m$-dNK did not affect the sensitivity of the cells to the nucleoside analogs or the efficiency of bystander cell killing. We also investigated the influence of Tan IIA on the bystander effect of cells expressing $D m$-dNK, and whether there were any statistical differences between the $D m$-dNK expression in nuclei and in mitochondria. Furthermore, the present study, examined the relationship between Tan IIA and the expressions of $\mathrm{Cx} 43$ and $\mathrm{Cx} 26$ in order to elucidate the primary mechanism of the anticancer activities of Tan IIA.

\section{Materials and methods}

Cell culture. RetroPack PT67 packaging cells (Clontech, Palo Alto, CA, USA) and thymidine kinase (TK)-deficient osteosarcoma cells (a gift from Professor Jan Balzarini, Rega Institute, Leuven, Belgium) were cultured in Dulbecco's modified Eagle's medium (DMEM) supplemented with $10 \%(\mathrm{v} / \mathrm{v})$ fetal calf serum (Gibco-BRL, Gaithersburg, MD, USA), 100 U/ml penicillin, and $0.1 \mathrm{mg} / \mathrm{ml}$ streptomycin. Cells were grown at $37^{\circ} \mathrm{C}$ in a humidified incubator with a gas phase of $5 \% \mathrm{CO}_{2}$.

Construction of retroviral vectors and subcellular localization of $D m-d N K$. We used the pLEGFP-N1 retroviral vector (Clontech) to express the $D m$-dNK cDNA in fusion with the green fluorescent protein (dNK-GFP) (Fig. 1). The pLEGFP-N1 with wild-type Dm-dNK was constructed as previously described (10) and cloned into the XhoI-BamHI site of the pLEGFP-N1 vector. The cDNA sequence encoding the 31-amino acid N-terminal mitochondrial import signal of cytochrome $c$ oxidase subunit VIII was cloned upstream of $D m$-dNK, and C-terminal deletions (20 amino acids deleted) were made using the primer 5'-TCGTCGACTTATG GATGGCGTCGAATATGCTGCT-3'. Plasmids were purified using the NucleoBond plasmid purification kit (Clontech). The DNA sequences of the constructed plasmids were verified using an ABI 310 automated DNA sequencer (Applied Biosystems, Foster City, CA, USA). The constructed pLEGFP-N1 plasmids were transfected into the PT67 packaging cells using Lipofectamine (Life Technologies, Inc., Grand Island, NY, USA) according to the protocol provided by the supplier. The medium from the transfected cells was collected $48 \mathrm{~h}$ after transfection, filtered through a $0.45-\mathrm{mm}$ filter, and diluted 2-fold with fresh medium. The osteosarcoma cells were incubated with the virus-containing medium for $48 \mathrm{~h}$ and cultured continuously for 3 weeks in the presence of $1.0 \mathrm{mg} / \mathrm{ml}$ Geneticin (Gibco-BRL). The cell nuclei were counterstained with 4',6-diamidino-2-phenylindole (DAPI) and the mitochondria were counterstained with MitoTracker (Invitrogen, Ltd., Paisley, UK). GFP, DAPI and MitoTracker fluorescence was observed using a Nikon Eclipse E600 microscope (Nikon, Tokyo, Japan) equipped with a SPOT RT digital camera (Diagnostic Instruments, Inc., Sterling Heights, MI, USA).

Western blot analysis and enzymatic assays. Nuclear extracts were prepared as previously described (8). The mitochondrial fractions were isolated from transduced osteosarcoma cells as previously described (28) by differential centrifugation in lysis buffer $(0.3 \mathrm{M}$ mannitol, $0.1 \%$ bovine serum albumin, 2 mM EDTA, 10 mM HEPES, pH 7.4). After cell homogenization with a glass homogenizer, the suspension was centrifuged for $10 \mathrm{~min}$ at $1,000 \mathrm{x} \mathrm{g}$ at $4^{\circ} \mathrm{C}$ (the supernatant contained cellular fractions). The supernatant was centrifuged again at $14,000 \mathrm{x} \mathrm{g}$ for $15 \mathrm{~min}$ at $4^{\circ} \mathrm{C}$. To pellet the mitochondrial fraction, the supernatant was ultra-centrifuged at $100,000 \mathrm{x} \mathrm{g}$ for $60 \mathrm{~min}$. The concentration of the extracted protein was measured using a BCA protein assay (Kaiji, China). The protein extracts were separated on a $12 \%$ SDS-PAGE gel and electro-transferred to a nitrocellulose membrane. The membrane was probed for $1 \mathrm{~h}$ at room temperature with a polyclonal anti-GFP antibody (Invitrogen, Ltd.). Binding of the primary antibody was detected using a secondary mouse anti-rabbit immunoglobulin (Ig) conjugated to horseradish peroxidase (Amersham, Arlington Heights, IL, USA). ECL reagents were used to detect the signals according to the manufacturer's instructions (Amersham). The enzymatic assays were performed as previously described (8). For the reaction, $3 \mathrm{mM}$ [methyl-3H]dThd (Moravek Biochem, Burlington, Ontario Canada) and $2 \mathrm{mM}$ unlabeled dThd (Sigma-Aldrich, Beijing, China) were used.

For the analysis of connexin expression, cells were seeded at $1 \times 10^{4}$ cells/well in 6 -well plates. After $24 \mathrm{~h}$, the medium was removed and replaced with complete medium containing Tan IIA (Xi'an Guanyu Bio-tech Co., Ltd., China) at 0, 5, 10 and $20 \mu \mathrm{M}$, respectively. After 3 days, cells were washed twice with PBS and lysed in RIPA buffer (8\% SDS, $0.25 \mathrm{~m}$ Tris- $\mathrm{HCl}$, $\mathrm{pH} 6.8,1 \mathrm{mM}$ phenylmethylsulfonyl fluoride, $1.0 \mathrm{mg} / \mathrm{ml}$ leupeptin, and $10 \mathrm{mg} / \mathrm{ml}$ aprotinin). Total cell extracts were separated on $10 \%$ SDS-PAGE gels. Then western blot analyses were performed using anti-Cx43 (71-0700) and anti-Cx26 (CX-12H10) (both from Zymed, San Francisco, CA, USA) or anti-actin (ms-1295-po; NeoMarkers, Fremont, CA, USA) antibodies. 

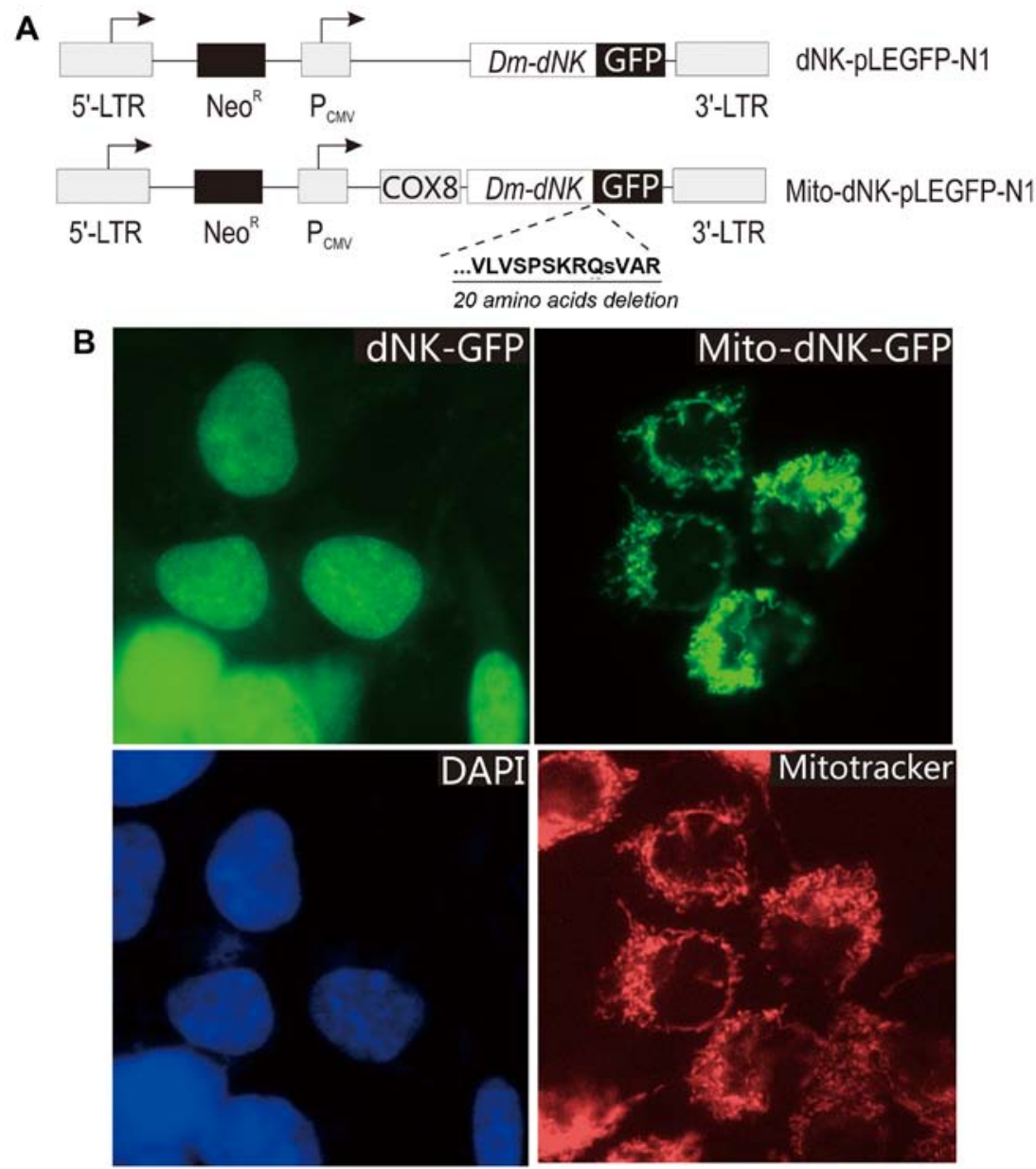

Figure 1. Expression of $\mathrm{Dm}$-dNK in the nuclei or mitochondria of osteosarcoma cells. (A) Retroviral vector construct harboring the wild-type nuclear $\mathrm{Dm}$-dNK (dNK-GFP) or the mitochondrial-targeted mutant mito-dNK-GFP. (B) Fluorescence microscopy of osteosarcoma cells transduced with the retroviruses. The GFP fluorescent signal and DAPI nuclear counterstain showed that the wild-type dNK-GFP was located in the nuclei, and the GFP fluorescent signal and Mitotracker counterstain showed that the mutant mito-dNK-GFP was expressed in the mitochondria. COX8, cytochrome $c$ oxidase subunit VIII mitochondrial targeting sequence; $\mathrm{P}_{\mathrm{CMV}}$, cytomegalovirus promoter; LTR, long terminal repeats; $\mathrm{Neo}^{\mathrm{R}}$, neomycin resistance gene; Dm-dNK, Drosophila melanogaster multi-substrate deoxyribonucleoside kinase.

Cell proliferation and bystander killing assays. araT was obtained from Lilly Research Laboratories. BVDU was a gift from Professor Jan Balzarini (Rega Institute, Leuven, Belgium). Cells were plated at 3,000 cells/well in 96-well plates. Nucleoside analogs were added $24 \mathrm{~h}$ after plating and the medium containing the nucleoside analogs was changed once during the 4-day incubation. Cell survival was assayed using a 3-(4,5-dimethylthiazol-2-yl)-2,5-diphenyltetrazolium bromide (MTT) assay (Boehringer Mannheim, Welwyn Garden City, UK) after 4 days of drug exposure. Each experiment was performed in triplicate.

The assay for bystander cell killing was performed as previously described (9). Tumor cells expressing $D m$-dNK were mixed at different ratios with their respective parental cell lines. To promote cell contacts, the mixed cells were plated in 24-well plates at $3 \times 10^{5}$ cells/well. The following day, confluent cells were treated with BVDU. After $24 \mathrm{~h}$ of incubation, cells were trypsinized and a 1:100 dilution of the cells was distributed into 96-well plates in five replicates. Cells were cultured subsequently in the presence of BVDU for 2-3 days until the cells without BVDU treatment reached confluence. Cell survival was determined as described above. Each experiment was performed in triplicate.
Effects of Tan IIA and BVDU on the growth of mixed cells. Cells expressing dNK-GFP or mito-dNK-GFP were mixed at a ratio of 1:9 with untransduced cells. For the measurement of cell viability, the mixtures were seeded at $3 \times 10^{3}$ cells/well in 96-well plates. After $24 \mathrm{~h}$, the medium was removed and replaced with complete medium with or without Tan IIA (5, 10,20 and $40 \mu \mathrm{M}$ ) for another $24 \mathrm{~h}$. Cells were incubated with BVDU $(0.1 \mu \mathrm{M})$ for $48 \mathrm{~h}$ in the presence of Tan IIA. Then MTT analysis was performed as described above. Each assay was repeated 3 times.

Statistical analysis. Data are expressed as the mean value \pm standard deviation. All experiments were performed in triplicate. All statistical analyses were performed using SPSS version 11.0. Comparisons among all groups were performed with one-way analysis of variance (ANOVA) and the Student-Newman-Keuls method. Statistical significance was indicated by a p-value $<0.05$.

\section{Results}

Expression of Dm-dNK in nuclei or mitochondria in a cancer cell line. To study the effects of phosphorylated nucleoside 

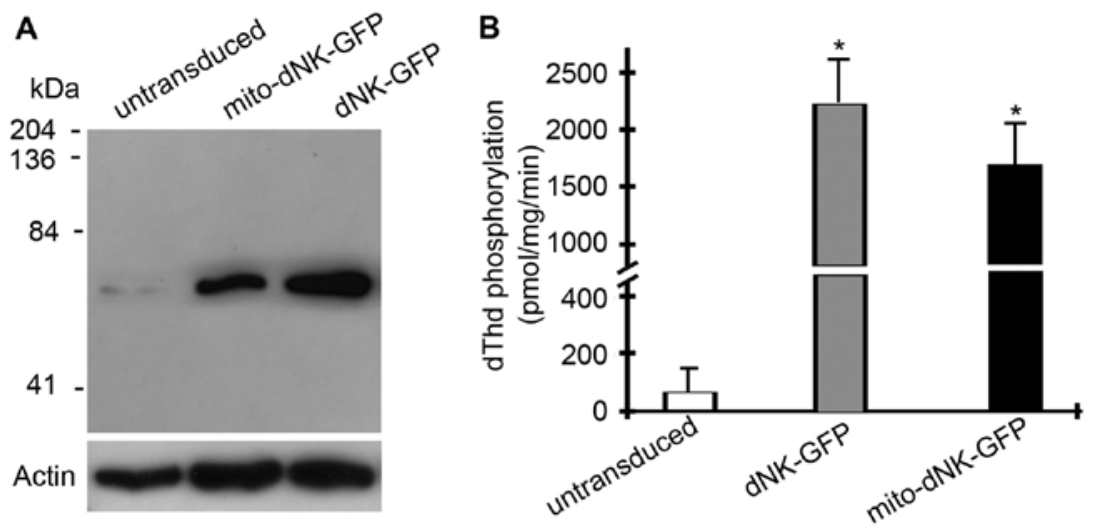

Figure 2. Expression and activities of dNK-GFP/Mito-dNK-GFP in the transduced cells. (A) Western blot analysis of cell extracts and mitochondrial fractions with an anti-GFP antibody. (B) $D m$-dNK activity, determined as dThd phosphorylation, in crude extracts of the untransduced and transduced osteosarcoma cells ("p<0.01). Dm-dNK, Drosophila melanogaster multi-substrate deoxyribonucleoside kinase.
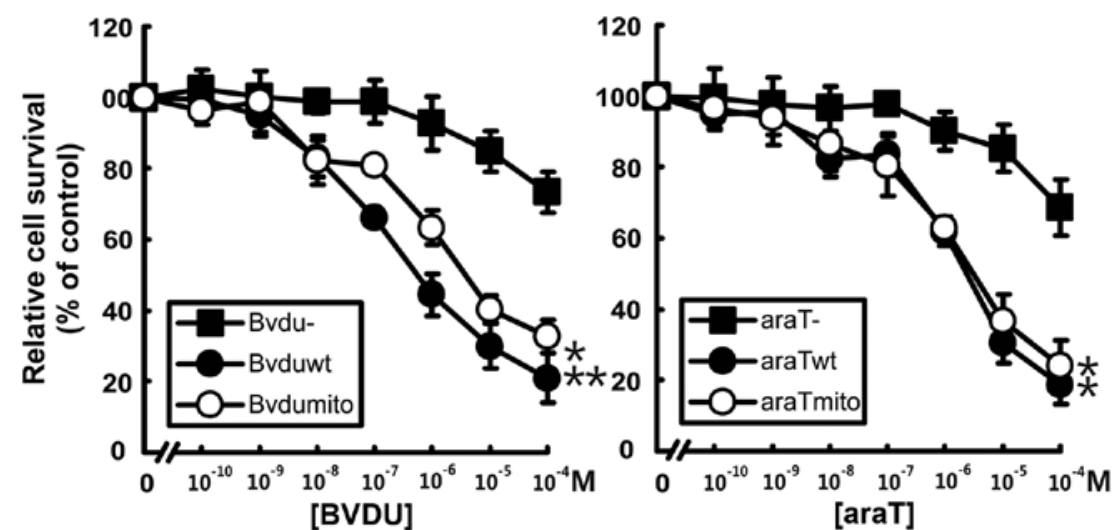

Figure 3. Sensitivities of untransduced osteosarcoma cells $(\boldsymbol{\bullet})$ and the cells expressing wild-type (wt) dNK-GFP $(\bullet)$ or mitochondrial mito-dNK-GFP (O) to BVDU and araT ("p<0.01, $\left.{ }^{* *} \mathrm{p}<0.001\right)$. BVDU, (E)-5-(2-bromovinyl)-2'-deoxyuridine; araT, 1- $\beta$-D-arabinofuranosylthymine.

analogs in the mitochondria matrix and to compare the sensitivities of the cells to nucleoside analogs and the efficiency of bystander cell killing when the enzyme was located in either of the two subcellular compartments, we aimed to express $D m$-dNK targeted to the mitochondria in TK-deficient osteosarcoma cancer cell lines. We fused the mitochondrial import signal of cytochrome $c$ oxidase subunit VIII to the $\mathrm{N}$-terminus of $D m$-dNK. For easy visualization of the subcellular localization of the protein, we also fused GFP to the C-terminus of $D m$-dNK, as we did with wild-type $D m$-dNK using the pLEGFP-N1 retroviral vector.

To achieve this, vectors harboring replication deficient retroviral elements were constructed to express either the wild-type nuclear $D m$-dNK (dNK-GFP) or the mitochondrial targeted $D m$-dNK mutant (mito-dNK-GFP) (Fig. 1A). A TK-1-deficient osteosarcoma cell line was transduced with the recombinant retroviruses. After selection of stably transfected cells, 90\% of the cells exhibited green fluorescence (Fig. 1B). The cells transduced with the virus encoding dNK-GFP exhibited fluorescence in the nucleus, whereas the cells transduced with the virus encoding mito-dNK-GFP had a dotted fluorescence pattern. These results were further confirmed by counterstaining with DAPI and MitoTracker (Fig. 1B), respectively. A co-localization of GFP and DAPI fluorescence indicated that the protein was located in the nuclei, and a co-localization of GFP and MitoTracker fluorescence indicated that the protein was located in the mitochondria. Western blot analyses with anti-GFP antibodies also detected the dNK-GFP and mito-dNK-GFP fusion proteins $(60 \mathrm{kDa})$ in the nuclei and the mitochondria, respectively (Fig. 2A).

To measure the enzymatic activities of the $D m$-dNK-GFP fusion proteins, we assayed the dThd phosphorylation activities in the cell extracts (Fig. 2B). The dThd kinase activities increased $\sim 40$-fold in the cells expressing the nuclear dNK-GFP and $\sim 35$-fold in the cells expressing the mitochondrial mito-dNK-GFP, compared with the untransduced parent cells ( $\mathrm{p}<0.01)$. There was no significant difference in $D m$-dNK activity between the cells expressing dNK-GFP in the mitochondria or in the nuclei.

Nucleoside analog sensitivity and bystander cell killing. We determined the sensitivities of the transduced cells to the pyrimidine nucleoside analogs BVDU and araT (Fig. 3). The two $D m$-dNK-GFP-expressing osteosarcoma cell lines were more sensitive to the nucleoside analogs than untransduced cells. The cells expressing $D m$-dNK in either the nuclei or in the mitochondria exhibited 100- to 500 -fold lower $\mathrm{IC}_{50}$ values to araT and BVDU than the untransduced cells $(\mathrm{p}<0.01)$. There 

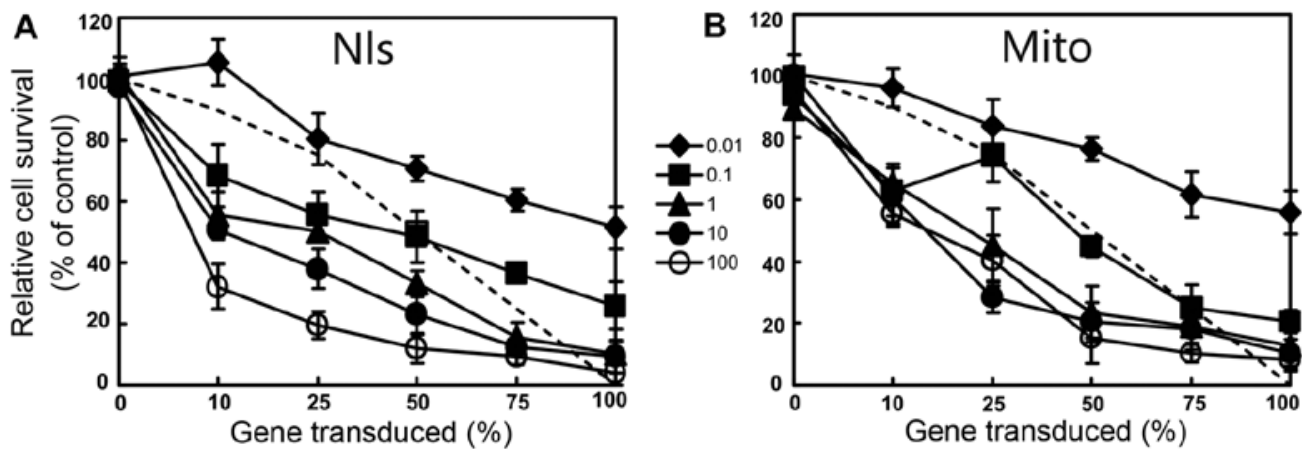

Figure 4. In vitro bystander cell killing of untransduced osteosarcoma cells mixed in different ratios with dNK-GFP (A) or mito-dNK-GFP (B). The cells were incubated with $0.01,0.1,1,10$, or $100 \mu \mathrm{M}$ BVDU. The cell survival (mean of five experiments \pm standard deviation) is expressed relative to cells incubated without BVDU. Dashed line indicates the calculated cell survival if only Dm-dNK-expressing cells have been killed and if no bystander effect has occurred. BVDU, (E)-5-(2-bromovinyl)-2'-deoxyuridine.

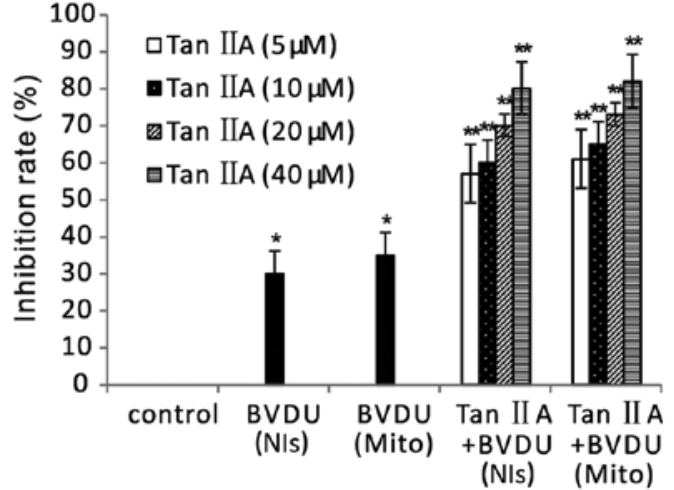

Figure 5. Tan IIA in combination with BVDU causes greater growth inhibition of the cells expressing dNK-GFP or mito-dNK-GFP mixed at the ratio of $10 \%$ with untransduced cells. The mixture of cells was untreated or treated with Tan IIA $(5,10,20$ and $40 \mu \mathrm{M})$ for $24 \mathrm{~h}$. Then they were cultured with BVDU $(0.1 \mu \mathrm{M})$ for $48 \mathrm{~h}$ followed by MTT assay. ${ }^{*} \mathrm{p}<0.05,{ }^{* *} \mathrm{p}<0.01$ compared with control or BVDU alone. Tan IIA, tanshinone IIA; BVDU, (E)-5-(2-bromovinyl)-2'-deoxyuridine.

were no differences in sensitivity to the nucleoside analogs between the cells expressing $D m$-dNK in the nuclei or in the mitochondria.

The cells expressing dNK-GFP or mito-dNK-GFP were mixed at different ratios $(0,10,25,50,75$ and $100 \%)$ with the untransduced cells. BVDU was added to the mixed cells at concentrations from 0.01 to $100 \mu \mathrm{M}$ and cells were incubated for 4 days. A bystander effect was observed in the osteosarcoma cell lines expressing dNK-GFP or mito-dNK-GFP (Fig. 4). For example, we found that $10 \%$ of the cells expressing dNK-GFP and $25 \%$ of the cells expressing mito-dNK-GFP induced $70 \%$ cell death in the presence of $100 \mu \mathrm{M}$ BVDU (Fig. 4). There were no differences in bystander killing between the cells expressing $D m$-dNK in the nuclei or in the mitochondria, in the presence of BVDU.

Tan IIA enhances the bystander effect by increasing the expression of Cx43 and Cx26 in TK-deficient osteosarcoma cell lines. Previous studies have shown that Tan IIA possessed not only anti-inflammatory (15) and antioxidant properties (29), but also anticancer activities through its influence on GJIC, in cell experiments and animal carcinogenesis models $(16,17)$.
A

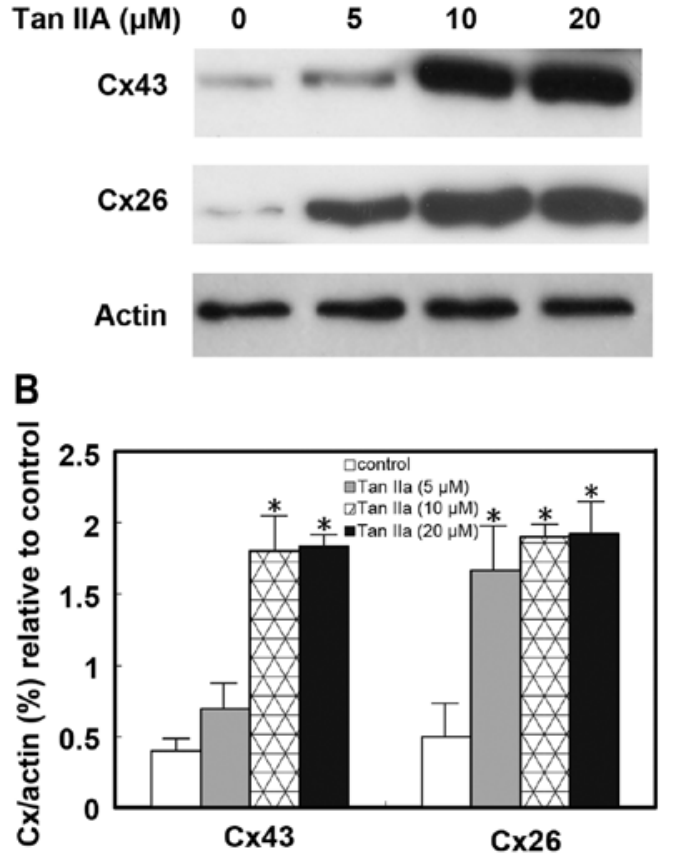

Figure 6. Tan IIA treatment of osteosarcoma cells results in the upregulation of Cx43 and Cx26 proteins. Cells were treated with Tan IIA $(0,5,10$ or $20 \mathrm{mM}$ ) for $72 \mathrm{~h}$. (B) Densitometric quantitation of independent western blot experiments performed as in (A). Western blot results from Cx43 and Cx26 were scanned and quantitated using Image Quant 5.0 software. Values are mean arbitrary units \pm SE from three independent experiments $\left({ }^{*} \mathrm{p}<0.05\right)$ Tan IIA, tanshinone IIA

To confirm the bystander effect of Tan IIA on cancer cells, we performed an MTT assay to assess the viability of a combination of cells expressing dNK-GFP or mito-dNK-GFP and untransduced cells at a ratio of 1:9. After BVDU treatment alone, there was a slight inhibition of viability, and there was no significant difference in the cells expressing $D m$-dNK in the nuclei and in the cells expressing $D m$-dNK in the mitochondria (Fig. 5). Tan IIA and BVDU treatment induced significantly greater inhibition of viability in mixed cells compared with BVDU treatment alone $(\mathrm{p}<0.01)$. There was no significant difference between the cells expressing dNK-GFP and the cells expressing mito-dNK-GFP. 
To further ascertain the effect of Tan IIA on GJIC in TK-deficient osteosarcoma cells, we examined the influence of Tan IIA on the expression of connexins Cx43 and Cx26. Cells were cultured and treated with Tan IIA at different concentrations $(0,5,10$ and $20 \mathrm{mM})$ for $72 \mathrm{~h}$. The results of western blot analysis showed that the expression levels of $\mathrm{Cx} 43$ and Cx26 were significantly upregulated (p<0.05; Fig. 6). Thus the exposure of TK-deficient osteosarcoma cells to Tan IIA upregulated the expression of the important gap junction proteins $\mathrm{Cx} 43$ and Cx26.

\section{Discussion}

$D m$-dNK localizes to cell nuclei when expressed in human cell lines and this localization is mediated by a nuclear localization signal in the C-terminal region of the protein (8). It is likely that the nuclear localization signal traps the protein in the nuclei and prevents it from importing to the mitochondria (30). We used a mutant and fused the mitochondrial import signal to its $\mathrm{N}$-terminus and GFP to the C-terminus (mito-dNK-GFP), and deleted $20 \mathrm{C}$-terminal amino acid residues that contained the nuclear localization signal. An osteosarcoma cell line stably expressing the mitochondrial $D m$-dNK was generated and exhibited green fluorescence. Our data showed that Dm-dNK retained its activity when expressed in the mitochondria, and that the cell lines expressing the enzyme exhibited increased sensitivity to the tested nucleoside analogs. These results were the same as those obtained with a mutant of $D m$-dNK generated by cloning a mitochondrial import signal before $D m$-dNK into the pEGFP-N1 vector but with a mutation of the arginine residue to serine in position 247 , to destroy the nuclear localization signal (30).

Mitochondrial DNA is replicated independently of nuclear DNA and the dNTP precursor pool is also separated $(31,32)$ due to the inner mitochondrial membrane between the mitochondrial matrix and the cytosol (33). Studies suggest that the majority of deoxyribonucleotides in the mitochondrial matrix may be trapped and are incorporated into mitochondrial DNA directly $(32,34)$. The enzymes involved in mitochondrial DNA replication differ from those catalyzing nuclear DNA replication, and several features of DNA replication in these two compartments are also different. Unlike the replication of nuclear DNA, mitochondrial DNA replication is independent of the cell cycle and lacks efficient repair mechanisms $(35,36)$. These features may be beneficial when the enzymes/nucleoside analogs involved in mitochondrial DNA replication are used in suicide gene therapies.

We previously found that there are no differences in enzyme activity, cellular sensitivity to nucleoside analogs, and bystander cell killing whether the enzyme is expressed in the cytosol or in the nucleus (10). In the present study, we showed that whether the $D m$-dNK-GFP was expressed in the nuclei or in the mitochondria also did not affect cellular sensitivity to cytotoxic nucleoside analogs or the bystander effect. Our data also showed that the cellular sensitivity to cytotoxic nucleoside analogs and to bystander cell killing were not dependent on the subcellular localization of the enzyme. However, previous results have suggested that due to the compartmentalized dNTP pools, nucleoside analogs phosphorylated in the mitochondrial matrix do not induce bystander cell killing (37). There are important differences between mitochondrial and nuclear DNA physiology. These include the non-S-phase restricted replication of mitochondrial DNA and the less efficient DNA repair systems in mitochondria compared with nuclear DNA replication $(35,36)$. These differences suggest that nucleoside analogs targeting nuclear or mitochondrial DNA may exhibit different pharmacological profiles. The nucleoside analogs may accordingly, primarily affect mitochondrial DNA. Bystander cell killing is mediated by the intercellular transport of phosphorylated nucleoside analogs via gap junctions in the cell membrane, and gap junctions may also exist in the inner mitochondrial membrane. An alternative explanation for this, is that a small amount of phosphorylated nucleoside analogs are transported from the mitochondrial matrix to the cytosol and nucleus. Previous studies have suggested that nucleoside analogs phosphorylated within the mitochondrial matrix are trapped and fail to be exported to the cytosol or nucleus $(31,34)$. Certain nucleosides and nucleoside analogs seem to be preferentially incorporated into both mitochondrial and nuclear DNA, and there is strong evidence supporting the communication between the mitochondrial and cytosolic/nuclear dNTP pools (38). We cannot presently distinguish between these mechanisms based on the results obtained. To develop novel treatment strategies, the cell culture model system described herein will be used to further study the pharmacological effects of nucleoside analog phosphorylation in the mitochondria.

Previous studies have shown that Tan IIA has anticancer activities and can influence the bystander effect, which is mediated by gap junctional intercellular communication (GJIC) $(16,17)$. We found that Tan IIA and BVDU significantly enhanced the bystander cell killing compared with BVDU treatment alone, and that there was no significant difference between cells expressing $D m$-dNK in nuclei or in mitochondria. Tan IIA was found to increase the expression of $\mathrm{Cx} 43$ by inhibiting the elevated miR-1 expression in ischemic and hypoxic cardiomyocytes $(18,19)$. We also found in this study that Tan IIA increased the expression of Cx43 and Cx26. The aberrant expression and downregulation of Cx43 and Cx26 may lead to the progression of various cancers (39-41). The present results revealed the primary mechanism involved in the enhanced effect of Tan IIA on the bystander effect in cells expressing $D m$-dNK in nuclei and mitochondria. Our study showed that a safe dosage of Tan IIA exhibited a strong cancer cell killing effect. However, further studies are needed to examine the substantial clinical benefit of Tan IIA for clinical practice in the future.

\section{Acknowledgements}

This study was supported by grants from the Hi-Tech Research Development Program of China (863 Program, 2006AA02Z493) and the National Natural Science Foundation of China (no. 81071900, 81172199 and 81272920).

\section{References}

1. Duarte S, Carle G, Faneca H, de Lima MC and Pierrefite-Carle V: Suicide gene therapy in cancer: Where do we stand now? Cancer Lett 324: 160-170, 2012 . 
2. Culver KW, Ram Z, Wallbridge S, Ishii H, Oldfield EH and Blaese RM: In vivo gene transfer with retroviral vector-producer cells for treatment of experimental brain tumors. Science 256: 1550-1552, 1992.

3. Moolten FL: Tumor chemosensitivity conferred by inserted herpes thymidine kinase genes: Paradigm for a prospective cancer control strategy. Cancer Res 46: 5276-5281, 1986.

4. Reardon JE: Herpes simplex virus type 1 and human DNA polymerase interactions with 2'-deoxyguanosine 5'-triphosphate analogues. Kinetics of incorporation into DNA and induction of inhibition. J Biol Chem 264: 19039-19044, 1989.

5. Dilber MS, Abedi MR, Christensson B, Björkstrand B, Kidder GM, Naus CC, Gahrton G and Smith CI: Gap junctions promote the bystander effect of herpes simplex virus thymidine kinase in vivo. Cancer Res 57: 1523-1528, 1997.

6. Freeman SM, Abboud CN, Whartenby KA, Packman $\mathrm{CH}$, Koeplin DS, Moolten FL and Abraham GN: The 'bystander effect': Tumor regression when a fraction of the tumor mass is genetically modified. Cancer Res 53: 5274-5283, 1993.

7. Ma S, Zhao L, Zhu Z, Liu Q, Xu H, Johansson M, Karlsson A and Zheng X: The multisubstrate deoxyribonucleoside kinase of Drosophila melanogaster as a therapeutic suicide gene of breast cancer cells. J Gene Med 13: 305-311, 2011.

8. Zheng X, Johansson M and Karlsson A: Retroviral transduction of cancer cell lines with the gene encoding Drosophila melanogaster multisubstrate deoxyribonucleoside kinase. J Biol Chem 275: 39125-39129, 2000.

9. Zheng X, Johansson M and Karlsson A: Bystander effects of cancer cell lines transduced with the multisubstrate deoxyribonucleoside kinase of Drosophila melanogaster and synergistic enhancement by hydroxyurea. Mol Pharmacol 60: 262-266, 2001

10. Zheng X, Johansson M and Karlsson A: Nucleoside analog cytotoxicity and bystander cell killing of cancer cells expressing Drosophila melanogaster deoxyribonucleoside kinase in the nucleus or cytosol. Biochem Biophys Res Commun 289: 229-233, 2001.

11. Zhu Z, Mao L, Zhao L, Sun Z, Wang Z, Xu H and Zheng X: Synergistic therapeutic effect in gastric cancer cells produced by oncolytic adenovirus encoding Drosophila melanogaster deoxyribonucleoside kinase. Cancer Biol Ther 11: 874-882, 2011

12. Johansson M, van Rompay AR, Degrève B, Balzarini J and Karlsson A: Cloning and characterization of the multisubstrate deoxyribonucleoside kinase of Drosophila melanogaster. J Biol Chem 274: 23814-23819, 1999.

13. Munch-Petersen B, Piskur J and Sondergaard L: Four deoxynucleoside kinase activities from Drosophila melanogaster are contained within a single monomeric enzyme, a new multifunctional deoxynucleoside kinase. J Biol Chem 273: 3926-3931, 1998.

14. Fish JM, Welchons DR, Kim YS, Lee SH, Ho WK and Antzelevitch C: Dimethyl lithospermate B, an extract of Danshen, suppresses arrhythmogenesis associated with the Brugada syndrome. Circulation 113: 1393-1400, 2006.

15. Yin X, Yin Y, Cao FL, Chen YF, Peng Y, Hou WG, Sun SK and Luo ZJ: Tanshinone IIA attenuates the inflammatory response and apoptosis after traumatic injury of the spinal cord in adult rats. PLoS One 7: e38381, 2012.

16. Liu F, Yu G, Wang G, Liu H, Wu X, Wang Q, Liu M, Liao K, Wu M, Cheng X, et al: An NQO1-initiated and p53-independent apoptotic pathway determines the anti-tumor effect of tanshinone IIA against non-small cell lung cancer. PLoS One 7: e42138, 2012.

17. Wang J, Wang X, Jiang S, Yuan S, Lin P, Zhang J, Lu Y, Wang Q, Xiong Z, Wu Y, et al: Growth inhibition and induction of apoptosis and differentiation of tanshinone IIA in human glioma cells. J Neurooncol 82: 11-21, 2007.

18. Zhang Y, Zhang L, Chu W, Wang B, Zhang J, Zhao M, Li X, Li B, Lu Y, Yang B, et al: Tanshinone IIA inhibits miR-1 expression through p38 MAPK signal pathway in post-infarction rat cardiomyocytes. Cell Physiol Biochem 26: 991-998, 2010.

19. Shan H, Li X, Pan Z, Zhang L, Cai B, Zhang Y, Xu C, Chu W, Qiao G, Li B, et al: Tanshinone IIA protects against sudden cardiac death induced by lethal arrhythmias via repression of microRNA-1. Br J Pharmacol 158: 1227-1235, 2009.

20. Söhl G and Willecke K: Gap junctions and the connexin protein family. Cardiovasc Res 62: 228-232, 2004.
21. Dbouk HA, Mroue RM, El-Sabban ME and Talhouk RS: Connexins: A myriad of functions extending beyond assembly of gap junction channels. Cell Commun Signal 7: 4, 2009.

22. Klein G: Cancer, apoptosis, and nonimmune surveillance. Cell Death Differ 11: 13-17, 2004.

23. Trosko JE and Ruch RJ: Cell-cell communication in carcinogenesis. Front Biosci 3: d208-d236, 1998.

24. Mesnil M, Piccoli C, Tiraby G, Willecke $\mathrm{K}$ and Yamasaki $\mathrm{H}$ : Bystander killing of cancer cells by herpes simplex virus thymidine kinase gene is mediated by connexins. Proc Natl Acad Sci USA 93: 1831-1835, 1996.

25. Elshami AA, Saavedra A, Zhang H, Kucharczuk JC, Spray DC, Fishman GI, Amin KM, Kaiser LR and Albelda SM: Gap junctions play a role in the 'bystander effect' of the herpes simplex virus thymidine kinase/ganciclovir system in vitro. Gene Ther 3: 85-92, 1996.

26. Wygoda MR, Wilson MR, Davis MA, Trosko JE, Rehemtulla A and Lawrence TS: Protection of herpes simplex virus thymidine kinase-transduced cells from ganciclovir-mediated cytotoxicity by bystander cells: The Good Samaritan effect. Cancer Res 57: 1699-1703, 1997.

27. Lawrence TS, Rehemtulla A, Ng EY, Wilson M, Trosko JE and Stetson PL: Preferential cytotoxicity of cells transduced with cytosine deaminase compared to bystander cells after treatment with 5-flucytosine. Cancer Res 58: 2588-2593, 1998.

28. Ljubkovic M, Marinovic J, Fuchs A, Bosnjak ZJ and Bienengraeber M: Targeted expression of Kir6.2 in mitochondria confers protection against hypoxic stress. J Physiol 577: 17-29, 2006.

29. Lin R, Wang WR, Liu JT, Yang GD and Han CJ: Protective effect of tanshinone IIA on human umbilical vein endothelial cell injured by hydrogen peroxide and its mechanism. J Ethnopharmacol 108: 217-222, 2006.

30. Solaroli N, Zheng X, Johansson M, Balzarini J and Karlsson A: Mitochondrial expression of the Drosophila melanogaster multisubstrate deoxyribonucleoside kinase. Mol Pharmacol 72: 1593-1598, 2007.

31. Bestwick RK, Moffett GL and Mathews CK: Selective expansion of mitochondrial nucleoside triphosphate pools in antimetabolite-treated HeLa cells. J Biol Chem 257: 9300-9304, 1982.

32. Zhu C, Johansson M and Karlsson A: Incorporation of nucleoside analogs into nuclear or mitochondrial DNA is determined by the intracellular phosphorylation site. J Biol Chem 275: 26727-26731, 2000.

33. Dolce V, Fiermonte G, Runswick MJ, Palmieri F and Walker JE: The human mitochondrial deoxynucleotide carrier and its role in the toxicity of nucleoside antivirals. Proc Natl Acad Sci USA 98: 2284-2288, 2001.

34. Berk AJ and Clayton DA: A genetically distinct thymidine kinase in mammalian mitochondria. Exclusive labeling of mitochondrial deoxyribonucleic acid. J Biol Chem 248: 2722-2729, 1973.

35. Bogenhagen D and Clayton DA: Mouse L cell mitochondrial DNA molecules are selected randomly for replication throughout the cell cycle. Cell 11: 719-727, 1977.

36. Bogenhagen DF: Repair of mtDNA in vertebrates. Am J Hum Genet 64: 1276-1281, 1999.

37. Sanda A, Zhu C, Johansson M and Karlsson A: Bystander effects of nucleoside analogs phosphorylated in the cytosol or mitochondria. Biochem Biophys Res Commun 287: 1163-1166, 2001.

38. Ferraro P, Nicolosi L, Bernardi P, Reichard P and Bianchi V: Mitochondrial deoxynucleotide pool sizes in mouse liver and evidence for a transport mechanism for thymidine monophosphate. Proc Natl Acad Sci USA 103: 18586-18591, 2006

39. Ezumi K, Yamamoto $H$, Murata K, Higashiyama M, Damdinsuren B, Nakamura Y, Kyo N, Okami J, Ngan CY, Takemasa I, et al: Aberrant expression of connexin 26 is associated with lung metastasis of colorectal cancer. Clin Cancer Res 14: 677-684, 2008.

40. Ozawa H, Matsunaga T, Kamiya K, Tokumaru Y, Fujii M, Tomita $\mathrm{T}$ and Ogawa K: Decreased expression of connexin-30 and aberrant expression of connexin-26 in human head and neck cancer. Anticancer Res 27: 2189-2195, 2007.

41. Chen Y, Hühn D, Knösel T, Pacyna-Gengelbach M, Deutschmann N and Petersen I: Downregulation of connexin 26 in human lung cancer is related to promoter methylation. Int $\mathrm{J}$ Cancer 113: 14-21, 2005. 